\title{
Role of Intraoperative Frozen Section Diagnosis in the Management of Patients with CNS Lesions: Evaluation of 60 Consecutive Intraoperative Frozen Sections from 1991 to 1993
}

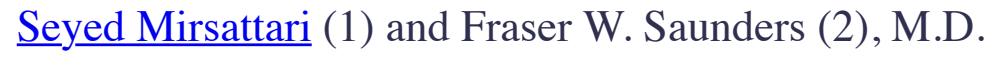

To whom correspondence should be addressed:

1. Faculty of Medicine

University of Ottawa, ON

Canada K1H 8M5

2. Division of Neurosurgery at the Kingston General Hospital and Queen's University in Kingston, Ontario

\begin{abstract}
To determine the role of intraoperative frozen sections (FSs) in the management of patients with central nervous system (CNS) lesions, 60 consecutive intraoperative clinical diagnoses of CNS lesions were presented and compared with concomitantly obtained FS diagnoses. Clinical diagnoses were established by history, physical examination, imaging techniques, and gross appearance of the abnormal tissue in situ. Tissue samples were obtained intraoperatively and processed for FS diagnoses. The findings of the FS diagnoses were reported to the operating room and compared with the clinical diagnoses. The remaining biopsy samples were used to prepare paraffin-embedded tissue sections from which the definitive diagnoses were made. Comparison of the clinical and FS diagnoses, using paraffin-embedded tissue as the true diagnosis, shows that FS diagnosis has a limited contribution to intraoperative patient management by the neurosurgeon. The rate of diagnostic failures between the two techniques was very similar; clinical diagnoses and FSs were misinterpreted in 12 and 11 of the 60 cases, respectively. Compared to a clinical diagnosis, the intraoperative FS technique provided no significant improvement in diagnosis and management; it altered the intraoperative management of the patients in 2 of 60 cases.
\end{abstract}

\section{INTRODUCTION}

Rapid histological diagnosis of neurosurgical biopsy material has always been of prime importance to the neurosurgeon (1). It is believed that preparations of histological sections, in which tissue structures are well preserved, may provide a more rapid and accurate diagnosis (2). Frozen section (FS), refers to a technique in which a section is cut by a cryostat from tissue that has been frozen. FS preserves the cellular detail and can provide rapid microscopic diagnosis of central nervous system (CNS) lesions $(3,4)$. Consequently, the FS 
technique, either alone or together with smear or imprint preparations, has become a routine method in neurosurgery (5-7). It is assumed that FS usually provides an immediate tissue diagnosis to guide the neurosurgeon in making clinically relevant intraoperative decisions.

Frequently, FS confirms the presence of a neoplasm and can determine its type. Once this is known, it can be established whether the neoplasm is benign or malignant, primary or metastatic, or of a type that lends itself to total extirpation. FS technique also provides information about the quality of the operative specimen and whether it should be studied further (3). However, accurate identification of the lesion should be made in the context of the patient's age, sex, neurologic signs, rate of symptom progression, anatomical location of the lesion, and radiographic and gross operative features (8). With the advances made in radiologic imaging techniques in recent years, the clinical pre- and intraoperative diagnosis has improved in degree of accuracy to the extent that it has become comparable to FS diagnosis.

Traditionally, almost all CNS lesions are biopsied and sent to the neuropathology laboratory for diagnosis by FS. However, the utility of this routine method has not been adequately evaluated in the literature. The objectives of this study were (i) to determine the actual role of FS diagnoses in guiding the neurosurgeon during the operation, and (ii) to compare FS diagnosis with the diagnosis established by the neurosurgeon after examining the gross appearance of the lesion intraoperatively. This study evaluated how often FS altered the diagnosis and intraoperative management of patients with CNS lesions.

\section{METHODS}

This was a study of 60 consecutive cases of intraoperative consultations in which FS preparations were compared to clinical diagnoses of the same cases using clinical history, physical examination, radiographic techniques, and intraoperative gross appearance of the abnormal tissue. Clinical diagnoses of various CNS lesions were examined along with their corresponding frozen and permanent histological sections. The study covered a period of 16 months, from November 1991 to March 1993. The clinical diagnoses were made preand intraoperatively; they were initiated preoperatively and the neurosurgeon made an independent intraoperative decision after examining the gross appearance of the abnormal tissue in situ. The results were recorded before the FS samples were examined. Surgical procedures were performed in the neurosurgical operating rooms of the Kingston General Hospital (KGH). A limited number of the patients (two cases) had undergone Computerized Tomography (CT) scan-directed stereotaxic biopsies.

FSs from one or more tissue specimens were prepared in the neuropathology laboratory close to the operating room. Each specimen was rapidly transported in saline from the operating room to the laboratory in a smooth, clean container. To minimize ice crystal formation, the specimen was mounted and immersed in isopentane to allow rapid freezing. Serial sections, 6 microns thick, were cut in a cryostat at -20ûC. Sections were then stained with routine hematoxylin and eosin (H\&E) (9). This FS preparation technique is similar to methods described in the literature $(1,10)$. To capture the cytologic details of tissue that might have been lost or artifacts that might have been gained during the FS process, imprint preparations were performed in most cases before processing the tissue for FS. A glass slide was placed against the tissue with moderate pressure and then immersed in a staining solution comprised of 1 part 37\% formaldehyde in 3 parts $95 \%$ ethanol.

After a few seconds the slide was washed in tap water and examined under the microscope. The result of the first FS specimen was reported to the operating room in approximately 15-20 minutes and compared against the clinical diagnosis. The two diagnoses and the effect of FS diagnosis on intraoperative management of each case were documented. In some cases, a diagnosis was already possible at this point. If the FS material proved to be non-diagnostic, further specimens were processed or additional ones were requested by the neurosurgeon for permanent sections.

The remainder of the tissue was embedded in paraffin and subsequently sectioned in order to establish a definitive histological diagnosis (9). Small blocks of formalin-fixed tissue were dehydrated and fixed in 
paraffin to provide a rigid matrix for cutting sections. H\&E was used for routine staining; additional stains and immunohistochemical methods were used, as required, depending on the case. Results of paraffinembedded sections were available within 24 to 48 hours. Since permanent sections prepared according to this protocol provide the best material for histologic diagnosis (3), they were used as the basis for the conclusive diagnosis by the pathology.

Generally, most patients had one or more imaging studies, including CT scan, myelogram, plain X-ray, tomogram, and angiogram, as part of the preoperative work-up. Only a limited number of patients had MRI because of limited access to this service at the time of this study.

\section{RESULTS}

Pertinent information analyzed from each of the 60 patients operated on at the KGH during the study period included location of the CNS lesion, clinical and FS diagnoses, discrepancy between the two techniques, effect of FS on the intraoperative management of the patient, and final tissue diagnoses. The topographic distribution of the cases analyzed showed that in 35 out of the 60 cases the masses were localized in the cerebral hemisphere. Of the remaining cases, tumors were located in the spinal cord region in 8 cases, the sellar-pituitary region in 6 cases, cerebellum in 4 cases, and cranial nerves in 3 cases. Thalamic, cerebellopontine angle, base of the skull, and orbital masses each accounted for single cases.

Brain tumor classification and malignancy grading used in the histological diagnoses corresponded with the guidelines of the WHO (11). Among the 60 cases, there were 23 gliomas (5 astrocytomas, 15 glioblastomas multiforme, and 3 ependymomas). Non-glial tumors accounted for 35 of the cases. Among these, there were 10 metastases, 7 pituitary adenomas, 6 meningiomas, and 1 multiple myeloma. Cerebral infarct and granulomatous inflammation each accounted for one case.

The intraoperative FS diagnosis and clinical diagnosis were compared with the postoperative paraffin embedded sections (definitive diagnosis) in all of these cases (Table 1). Clinical diagnoses coincided with the paraffin-embedded diagnoses $80 \%$ of the time (48 of the 60 cases), whereas FS corresponded with the paraffin-embedded diagnoses $81.7 \%$ of the time (49 of the 60 cases). Patt et al. (2), Broggi et al. (12), and Kleihues and Volk (13) demonstrated a similar positive correlation (84\%) between intraoperative FS/smear preparations and postoperative immunohistochemistry/paraffin embedding. In addition, the FS confirmed the initial clinical diagnosis in 48 cases. FSs were misinterpreted in three cases, and were considered nondiagnostic for technical reasons in eight cases. Diagnostic errors and non-diagnostic studies were compiled and considered together as errors in diagnoses. Table 2 summarizes the 16 cases of misdiagnoses made by the FS and/or clinical procedure(s).

In 58 of the 60 cases, the FS did not affect the immediate intraoperative management of the patient. There were two cases (referred to as cases 1 and 2 in Table 2) which were clinically misdiagnosed and where FS significantly affected the intraoperative management by the neurosurgeon. Case 1 was a metastatic carcinoma of the posterior fossa which was clinically diagnosed as a schwannoma. Case 2 was a sub-ependymoma on the surface of the brain stem which was clinically diagnosed as an astrocytoma.

Among the 12 cases of clinical misdiagnoses, there was a predominance of posterior fossa lesions (four cases). Three lesions in this site were correctly diagnosed by FS. Histologically, medulloblastoma, ependymoma, astrocytoma, and hemangioblastoma were difficult to differentiate by clinical features; FS was useful in determining the nature of these malignancies.

In 10 cases, the error made in clinical diagnosis involved failure to classify the lesion correctly and did not affect the immediate management decision by the neurosurgeon. 


\section{DISCUSSION}

From the evaluation of the data presented, it may be concluded that the rate of diagnostic discrepancies between clinical diagnosis and FS diagnosis was comparable ( $81.7 \%$ and $80 \%$, respectively). In most cases, the error made in clinical diagnosis involved only a failure to classify the lesion correctly and had no effect on the immediate management decision on the part of the neurosurgeon. There were only two cases where significant errors were made and the immediate management of the patients were dictated by the FS diagnoses (Table 2). While the treatment of choice for a schwannoma (Case 1) is surgical excision, metastatic carcinoma is usually managed medically or by radiation therapy, with or without surgery, depending on the presentation of the tumor (14). In Case 2, the neurosurgeon, guided by the FS diagnosis, was more aggressive in removing as much of the tumor as possible; the goal of surgery in the management of subependymoma is maximal resection of the tumor (14).

Diagnostic errors by FS were non-diagnostic in 8 of the 11 cases, and only 3 cases were incorrectly diagnosed. Although there is a considerable difference between a diagnostic error and a non-diagnostic result, in this study we regarded both of them as errors in diagnosis. In both cases of misdiagnosis, FS had no effect on the intraoperative management of the patient. In some non-diagnostic cases, the problem was not due to an error in diagnosis, but rather due to the clinical or pathological techniques being inadequate to make a firm diagnosis. An incorrect FS diagnosis did not lead to an inappropriate management of the patient.

A larger patient population is required to better correlate the location of the lesion and the histological type to the frequency of diagnostic errors by clinical means. In this study, posterior fossa lesions accounted for the majority of diagnostic errors by clinical diagnosis (4 of 12 errors), and FS was useful in the correct diagnosis where there was a clinical error ( 3 of 4 cases). Histologically, medulloblastoma, ependymoma, astrocytoma, and hemangioblastoma were difficult to differentiate by clinical features, whereas FS was useful in determining the nature of the malignancy.

The findings of this study indicate that clinical diagnosis is sufficient, in the majority of cases, to manage a patient intraoperatively. If any doubt exists about the nature of the lesion, FS could be utilized since it is relatively inexpensive and takes little time to perform. FS does not usually interfere with the operation itself or increase its duration. If requested, FS should be used in association with the clinical diagnosis.

The accuracy of clinical diagnosis was similar to that of the FS. In most cases, if there was any doubt concerning the nature of the malignancy by clinical diagnosis, FS did not improve the diagnosis. This study showed that in 2 of 60 cases, significant errors were made in clinical diagnoses, such that an error in patient management might have occurred had FS diagnosis not been obtained. However, the significance of this error is uncertain and remains to be determined by further studies using current imaging techniques.

The decision whether FS should continue to be part of the routine intraoperative work-up in the management of patients with CNS lesions should depend on the centers that perform such neurosurgical operations. While it may be a vital training tool in a teaching hospital, it may appear futile in other centers. Hwang (10), Rosai (15), and Ackerman and Ramirez (16) have made similar conclusions, and have acknowledged that FS should only be requested for those instances in which an immediate therapeutic decision can be made.

This study suggests that although an intraoperative FS examination can contribute to the accurate diagnosis of CNS lesions, it alters patient management in only a few cases ( 2 of 60 cases in this study). History, physical examination, radiologic imaging techniques, and intraoperative gross appearance of the abnormal tissue usually allow the neurosurgeon to make an accurate management decision.

\section{ACKNOWLEDGEMENTS}


In preparing this paper, we have benefited greatly from the generous advice of our colleagues in the division of neurosurgery. Foremost among those to whom we are indebted are Drs. J. Marshall and F. Espinosa, who kindly participated in the design of the study and spent substantial amounts of their precious time in recording the results. In addition, we wish to offer many thanks to Drs. D.M. Robertson and J. Rossiter (Division of Pathology), who kindly provided us with the pathology report for every case.

\section{REFERENCES}

1. Tashiro K, Tsuru M. The rapid microscopical diagnosis of brain and spinal cord tumors by cryostat-cut frozen section. No Shinkei Geka. 1975;3(4):323-327.

2. Patt S, Weigel K, Zimmer C, et al. Experience with the intraoperative frozen section technique for stereotaxic brain tumor biopsies. Zentralblatt fur Pathologie 1991;137(1):35-40.

3. Chandrasoma P, Taylor CR. Concise pathology-First edition. California: Appleton \& Lange, $1991: 311$.

4. Scheithauer BW. Central nervous system and pituitary. In: Silva EG, Kraemer BB, editors. Intraoperative pathologic diagnosis: Frozen Section and other techniques. Los Angeles: Williams\&Wilkins, 1987:167-219.

5. Guarda LA. Intraoperative cytologic diagnosis: evaluation of 370 consecutive intraoperative cytologies. Diagnostic Cytopathology 1990;6(4):234-242.

6. Burger PC. Use of cytological preparations in the frozen section diagnosis of central nervous system neoplasia. American Journal of Surgical Pathology 1985;9(5):344-354.

7. Bloustein PA, Silverberg SG. Rapid cytological examination of surgical specimens. Pathology Annual 1977;2:251-278.

8. Burger PC, Vogel FS. Frozen section interpretation in surgical neuropathology: I. Intracranial lesions. American Journal of Surgical Pathology 1977;1(4):323-347.

9. Kingston General Hospital. Division of Anatomical Pathology. Histopathology laboratory, procedures manual and test costs. Kingston, 1993.

10. Hwang TS, Ham EK, Kim CW, Chi JG, Park SH. An evaluation of frozen section biopsy in 4434 cases. Journal of Korean Medical Science 1987;4(2):239-245.

11. Zulch KJ. Histological typing of tumours of the central nervous system. Geneva: World Health Organization, 1979.

12. Broggi G, et al. Diagnostic accuracy and multimodal approach in stereotactic biopsies of deep brain tumors. Acta Neurochirugica. Supplementum 1984;33:211-212.

13. Kleihues P, Volk B, Anagnostopoluos J, et al. Morphologic evaluation of stereotactic brain tumor biopsies. Acta Neurochirugica. Supplementum 1984;33:171-81.

14. Greenberg MS. Handbook of neurosurgery, Second Edition. Lakeland: Greenberg Graphics, 1991:536,566,576.

15. Rosai J. Ackerman's surgical pathology, 6th ed. Vol. 1. St. Louis: The Mosby Company, 1981:14-15. 
16. Ackerman LV, Ramirez GA. The indications for and limitations of frozen section diagnosis. British Journal of Surgery 1959;46:336-350, 1959.

\section{AUTHOR BIOGRAPHY}

Seyed Mirsattari is presently a fourth-year medical student at the University of Ottawa (Ottawa, Ontario). His work on the role of frozen sections in intraoperative diagnosis was conducted during his second year of medical education at the Division of Neurosurgery, Kingston General Hospital and Queen's University. He will be a first-year neurology resident at the University of Manitoba (Winnipeg, Manitoba) in the coming year. 